\title{
Efficient particle acceleration from HESS J1640-465 and the PeVatron candidate HESS J1641-463
}

\author{
Arnaud Marès* \\ Centre d'Etudes Nucleaires de Bordeaux-Gradignan (CNRS-IN2P3 / Université de Bordeaux), \\ 19 Chemin du Solarium, 33175 Gradignan, France \\ E-mail: maresecenbg.in2p3.fr

\section{Marie-Hélène Grondin} \\ Centre d'Etudes Nucleaires de Bordeaux-Gradignan (CNRS-IN2P3 / Université de Bordeaux), \\ 19 Chemin du Solarium, 33175 Gradignan, France \\ E-mail: grondinecenbg.in2p3.fr

\section{Marianne Lemoine-Goumard} \\ Centre d'Etudes Nucleaires de Bordeaux-Gradignan (CNRS-IN2P3 / Université de Bordeaux), \\ 19 Chemin du Solarium, 33175 Gradignan, France \\ E-mail: lemoinedcenbg.in2p3. fr \\ On behalf of the Fermi-Lat Collaboration ${ }^{\dagger}$
}

HESS J1640-465 and HESS J1641-463 are two neighboring gamma-ray sources located in the Galactic plane. Detected by both the High Energy Stereoscopic System (H.E.S.S.) and the FermiLarge Area Telescope (LAT), these sources might be very efficient particle accelerators, even up to the PeV in the case of HESS J1641-463.

Here, we present dedicated morphological and spectral analyses of these sources using 100 months of Fermi-LAT data. The new results help to unveil the nature of the sources and study their properties.

36th International Cosmic Ray Conference -ICRC2019-

July 24th - August 1st, 2019

Madison, WI, U.S.A.

\footnotetext{
* Speaker.

${ }^{\dagger}$ for collaboration list see PoS(ICRC2019)1177
} 


\section{Introduction}

There are only a few PeVatron candidates known in our Galaxy which might accelerate particles up to the knee of the cosmic-ray spectrum. HESS J1641-463, a gamma-ray source located in the Galactic plane and detected by H.E.S.S. above $4 \mathrm{TeV}$, is one of them. Characterized as a point source with a hard spectral index of $\Gamma \sim 2.1$ [1], HESS J1641-463 remains unidentified but is coincident with a radio SNR G338.5+0.1 and the dense HII region G338.4+0.0, with a distance estimate of $11 \mathrm{kpc}$ with $\mathrm{HI}$ absorption [2].

With an angular extent of only $0.07^{\circ}$ [3], HESS J1640-465, coincident with the composite SNR G338.3-0.0 [4] and the pulsar PSR J1640-4631 [5], was originally classified as an extremely powerful SNR. In this context, the escape of cosmic-ray protons accelerated by G338.3-0.0 colliding with the ambient dense gas would be able to produce the emission of HESS J1641-463, providing a self-consistent explanation for the gamma-ray emission of both sources [6].

Using 100 months of Fermi-LAT Pass 8 data, we analyzed these two sources from $200 \mathrm{MeV}$ to $1 \mathrm{TeV}$. Our extensive morphological and spectral analyses provide new constraints on the origin of the gamma-ray emission as well as the efficiency of these two H.E.S.S. sources to accelerate protons and contribute to the Galactic cosmic-ray flux around the knee.

\section{Fermi-LAT observations}

Successfully launched on 2008 June 11, the Large Area Telescope (LAT), aboard the Fermi Gamma-ray Space Telescope is an electron-positron pair conversion telescope sensitive to gamma rays from 0.2 to more than $300 \mathrm{GeV}$ [7].

The analyses described below take full advantage of the improvements provided by the Pass 8 data selection and event-level analysis [8]. The gamma-ray sources are observed with more statistics, thanks to the increase of the acceptance of $20 \%$ above $1 \mathrm{GeV}$. This new version of the data also makes it possible to better take into account the "ghost" events [9]. An improvement of the tracking algorithm also allows a better reconstruction of the direction of incidence of photons. Then, Pass 8 makes it possible to select the events according to their quality of reconstruction and the precision of their energy measurement.

\section{Data analysis}

The following analyses were performed using 100 months of Pass 8 Fermi-LAT data collected primarily in survey mode. We selected only events with energies greater than $200 \mathrm{MeV}$, excluding those coming from a zenith angle larger than $90^{\circ}$ to the detector axis to reduce contamination from the Earth limb [11]. Time intervals when Fermi was within the South Atlantic Anomaly were excluded. 
The model includes sources from the $4 \mathrm{FGL}$ catalog [12] up to $20^{\circ}$ and the new diffuse emission models for Galactic (gll_iem_v07.fits) and isotropic (iso_P8R3_SOURCE_V2_v1.txt) emission ${ }^{1}$.

These analyses are performed with the maximum-likelihood method [13] called gtlike ${ }^{2}$. We use the four PSF events-type in a summed-likelihood analysis within a region of interest of $15^{\circ} \times$ $15^{\circ}$ centered on HESS J1640-465, between $1 \mathrm{GeV}$ and $1 \mathrm{TeV}$ using 24 energy bins for the morphological analysis and between $200 \mathrm{MeV}$ and $1 \mathrm{TeV}$ using 30 energy bins for the spectral analysis.

\subsection{Morphology}

We first perform a spatial analysis above $1 \mathrm{GeV}$ using FermiPy [10] with a bin size of $0.04^{\circ}$. Spectral parameters are left free for the brightest sources and within a radius of $4^{\circ}$.

In order to better fit the observed emission, we have to consider additional sources in the model. First, we make a residual $\mathrm{TS}^{3}$ map of the region above $1 \mathrm{GeV}$ using the sources from the 4FGL source list. This sky map contains the TS value for a point source at each map location assuming a power-law with fixed photon index of 2 , thus giving a measure of the statistical significance for the detection of a gamma-ray source in excess of the background. When the TS of one of this source is above 16, we add it in the model. If the TS of these added sources are above 25 after fitting their prefactor and their index, we keep them in the final source model of the region. Nine sources have been found by this method.

A similar search is done above $200 \mathrm{MeV}$, with hotspots that have this time a TS higher than 25 in the residual TS map, which allows to add two more sources in the model of the region.

Figure 1 shows the residual TS map of the region centered on HESS J1640-465 using sources from the 4FGL source list before adding the sources found by FermiPy (see above) to the model. By considering these sources, the residual TS map is much cleaner and the fit quality is improved.

Using the updated source model, we use FermiPy in an iterative way in order to find the best position and seek for the extension of both sources. First, we localize both sources using a point source assumption. Then, we look for the extension of HESS J1640-465 and compute its position while the position of HESS J1641-463 remains the same. Then, we use the extension and position found for HESS J1640-465 to re-localize HESS J1641-463 and test its extension.

For the first time, we measure a significant extension for HESS J1640-465 using Fermi-LAT data (Table 2), modeling it as a $2 \mathrm{D}$ Gaussian with a width of $\sigma=\left(0.068^{\circ} \pm 0.013_{\text {stat }}^{\circ}\right)$ above $1 \mathrm{GeV}$ with a $\mathrm{TS}_{\text {ext }}{ }^{4}$ of 39 .

\footnotetext{
${ }^{1}$ Available on FSSC : https://fermi.gsfc.nasa.gov/ssc/data/access/lat/BackgroundModels.html

${ }^{2}$ ScienceTools: v11-07-00

${ }^{3}$ The Test Statistic is defined as twice the log-likelihood difference between the model with a putative additional source and the reference model. It follows a chi2 law with $\mathrm{N}$ (number of parameters associated to the additional source) degrees of freedom assuming the reference model is correct and the $\mathrm{N}$ parameters are non-degenerate.

${ }^{4} \mathrm{We}$ look at the improvement of the TS of the source when we compare the extended model to the point source model assuming a power-law spectral shape for the test.
} 


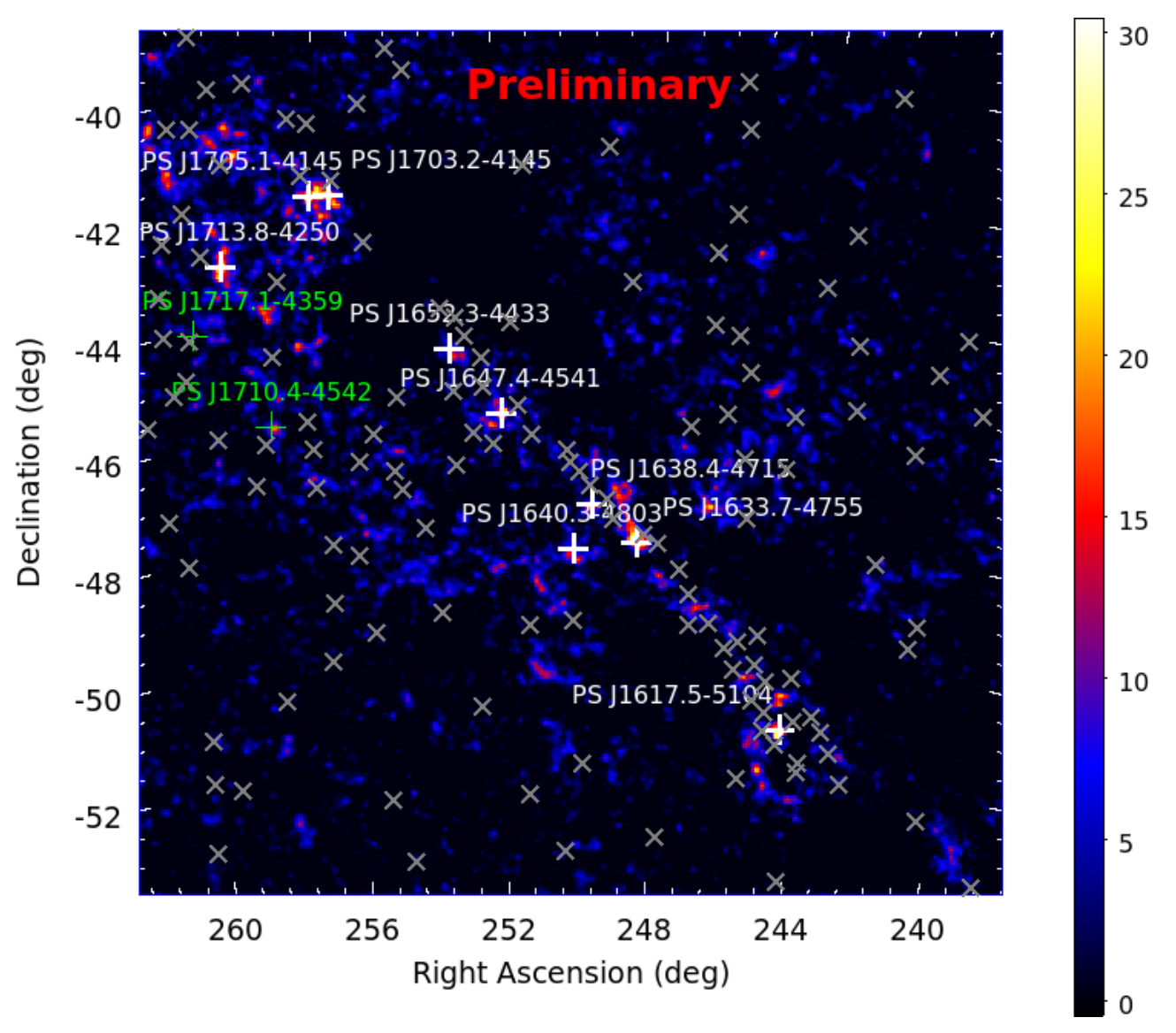

Figure 1: Residual TS map above $1 \mathrm{GeV}$ centered on HESS J1640-465. The grey crosses are the sources from the 4FGL catalog, the white crosses (and associated names) are the position of the sources added by FermiPy above $1 \mathrm{GeV}$ and the green one added above $200 \mathrm{MeV}$.

\begin{tabular}{|c|c|c|c|}
\hline \multicolumn{4}{|c|}{ Position and extension of HESS J1640-465 } \\
\hline & 4FGL & FermiPy & H.E.S.S. \\
\hline RA & $250.16^{\circ} \pm 0.02_{\text {stat }}^{\circ}$ & $250.18^{\circ} \pm 0.01_{\text {stat }}^{\circ}$ & $250.180^{\circ} \pm 0.004_{\text {stat }}^{\circ}$ \\
\hline Dec & $-46.55^{\circ} \pm 0.02_{\text {stat }}^{\circ}$ & $-46.56^{\circ} \pm 0.01_{\text {stat }}^{\circ}$ & $-46.530^{\circ} \pm 0.004_{\text {stat }}^{\circ}$ \\
\hline Extension & none & $\sigma=0.068^{\circ} \pm 0.013_{\text {stat }}^{\circ}$ & $\sigma=0.072^{\circ} \pm 0.003_{\text {stat }}^{\circ}$ \\
\hline \multicolumn{4}{|c|}{ HESS J1641-463 } \\
\hline & 4FGL & FermiPy & H.E.S.S. \\
\hline RA & $250.26^{\circ} \pm 0.02_{\text {stat }}^{\circ}$ & $250.25^{\circ} \pm 0.01_{\text {stat }}^{\circ}$ & $250.26^{\circ} \pm 0.01_{\text {stat }}^{\circ}$ \\
\hline Dec & $-46.32^{\circ} \pm 0.02_{\text {stat }}^{\circ}$ & $-46.29^{\circ} \pm 0.01_{\text {stat }}^{\circ}$ & $-46.30^{\circ} \pm 0.01_{\text {stat }}^{\circ}$ \\
\hline
\end{tabular}

Table 1: Position of sources

The 2D-Gaussian obtained with FermiPy is better than the H.E.S.S. template, but not in a significant way (Table 2) so we use the H.E.S.S. extension and position for the rest of the analysis. 


\begin{tabular}{ccc}
\multicolumn{3}{c}{ Morphology of HESS J1640-465 } \\
\hline \hline Model & Degree of Freedom (DoF) & $\mathrm{TS}_{\text {ext }}$ (point vs model $)$ \\
Point (estimated) & 4 & $/ / / /$ \\
2D Gauss & 5 & 39 \\
H.E.S.S. template & 2 & 37 \\
\hline
\end{tabular}

Table 2: Morphological test of HESS J1640-465

No significant extension is found for HESS J1641-463, we keep it as a point source with the position given in Table 1.

\subsection{Spectral analysis}

Using the best spatial model derived from the previous morphological analysis, we make a binned analysis with a combination of the four PSF event types in a joint likelihood function. The gamma-ray sources observed by the LAT above $200 \mathrm{MeV}$ are detected with a TS of 233 for HESS J1640-465 and 860 for HESS J1641-463.

We make a comparison of different spectral models for both sources (Table 3). The spectrum of HESS J1641-463 is described by a $\log$-parabola $\left(d N / d E \propto E^{-(\alpha+\beta \log (E / E b)}\right)$ with $\alpha=2.7 \pm$ $0.1_{\text {stat }} \pm 0.2_{\text {syst }}$ and a significant curvature ( $4 \sigma$ improvement with respect to a power-law model) of $\beta=0.1 \pm 0.03_{\text {stat }} \pm 0.1_{\text {syst }}$.

\begin{tabular}{cccc}
\multicolumn{4}{c}{ Model comparaison : 200 MeV $-1 \mathrm{TeV}$} \\
\hline \hline HESS J1640-4665 & HESS J1641-463 & DoF & $\Delta T S_{\text {model vs PLPL }}$ \\
\hline Power-law & Power-law & 4 & III/ \\
Power-law & Log-parabola & 5 & 18 \\
Log-parabola & Log-parabola & 6 & 26
\end{tabular}

Table 3: Spectral shape comparison

The spectrum of HESS J1640-465 is well described by a power-law $\left(\mathrm{dN} / \mathrm{dE} \propto E^{-\Gamma}\right)$ with a hard index of $\Gamma=1.8 \pm 0.1_{\text {stat }} \pm 0.2_{\text {syst }}$.

Figure 3 shows the best fit and the butterfly computed for both sources together with the Spectral Energy Distribution (SED) from the H.E.S.S. analysis at higher energy. There is a good connection betwen Fermi and H.E.S.S. data for HESS J1640-465. Concerning HESS J1641-463, it seems that Fermi and H.E.S.S. are detecting two distinct objects or two different mechanisms of radiation. The SED is pulsar-like in the LAT energy range. Fermi might see a pulsar while H.E.S.S. detects its associated nebula. 


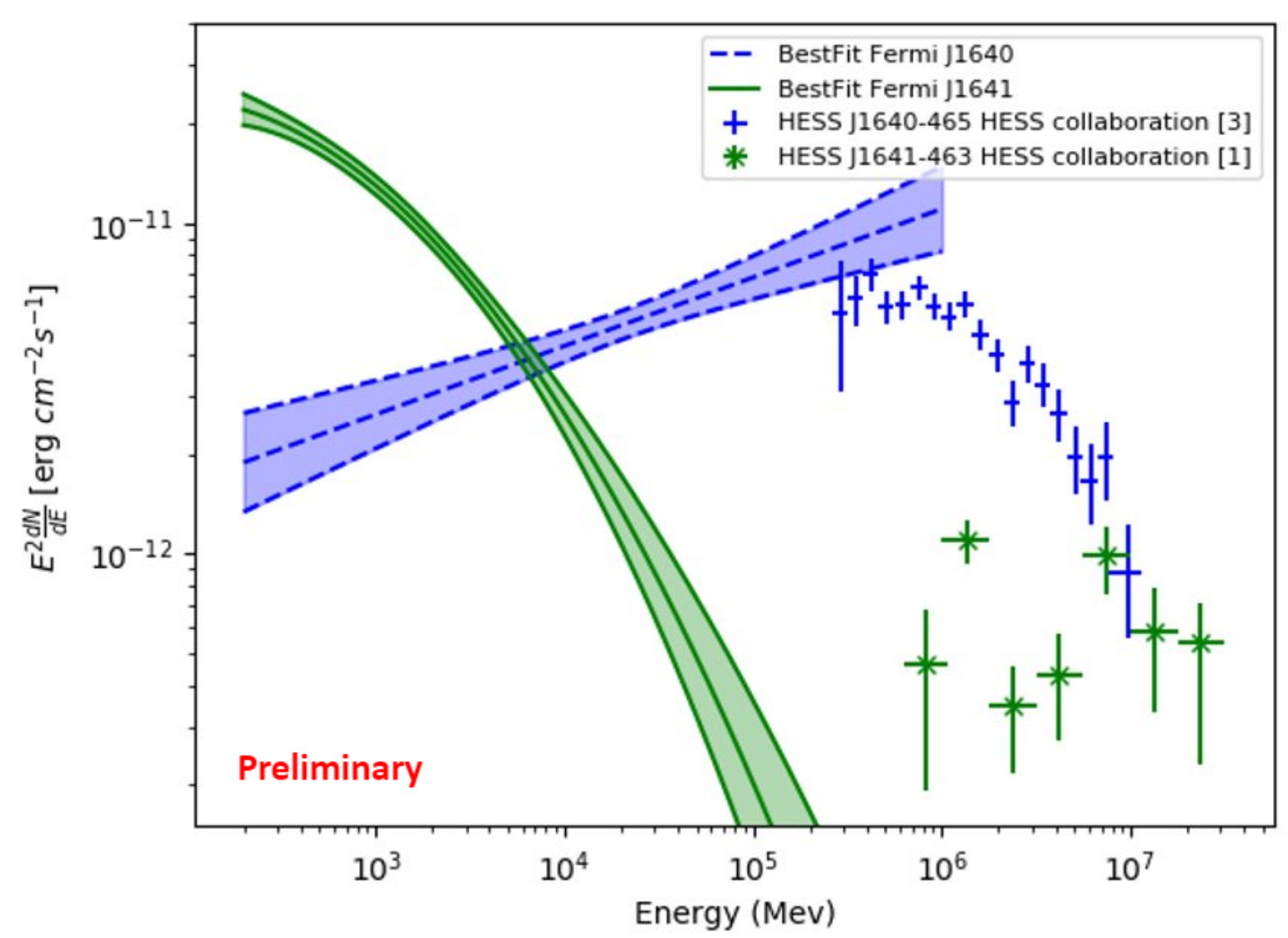

Figure 2: H.E.S.S. and Fermi spectra for HESS J1640-465 and HESS J1641-463

The integrated flux between $200 \mathrm{MeV}$ and $1 \mathrm{TeV}$ is $\left(4.44 \pm 0.15_{\text {stat }} \pm 0.33_{\text {syst }}\right) \times 10^{-11} \mathrm{erg}$ $\mathrm{cm}^{-2} \mathrm{~s}^{-1}$ for HESS J1640-465 and $\left(4.73 \pm 0.15_{\text {stat }} \pm 1.53_{\text {syst }}\right) \times 10^{-11} \mathrm{erg} \mathrm{cm}^{-2} \mathrm{~s}^{-1}$ for HESS J1641-463. The first error is statistical, while the second represents our estimate of systematic effects. The systematics taken into account are the uncertainties in our model of the Galactic diffuse emission, on the morphology of HESS J1640-465 and in our knowledge of the Fermi-LAT Instrument Response Function (IRFs).

The first uncertainty was estimated by comparing the results obtained using the standard Galactic diffuse model with the results based on eight alternative interstellar emission models as performed in [14]. The uncertainties on the morphology of HESS J1640-465 was estimated by making a comparison between the results obtained using the H.E.S.S. template and those using our best morphology using FermiPy. The third uncertainty is estimated by using modified IRFs whose effective areas bracket the nominal ones.

\section{Conclusion}

New morphological and spectral analyses are performed on the two high energy sources HESS J1640-465 and HESS J1641-463 using 100 months of Fermi LAT data. In this purpose, the new 
4FGL catalog released by the Fermi-LAT collaboration is used, together with the improved Pass 8 reconstruction and the new diffuse emission models.

For the first time, the significant extension of HESS J1640-465 is measured with the LAT data, with a hard index of $\Gamma=1.8$. Concerning HESS J1641-463, the spectrum looks like a pulsar one, with a significant curvature.

Search for pulsations and variability is currently on going in order to constrain the nature of HESS J1641-463 (pulsar/nebula, binary system or supernova remnant).

\section{Acknowledgement}

The Fermi-LAT Collaboration acknowledges generous ongoing support from a number of agencies and institutes that have supported both the development and the operation of the LAT as well as scientific data analysis. These include the National Aeronautics and Space Administration and the Department of Energy in the United States, the Commissariat à l'Énergie Atomique and the Centre National de la Recherche Scientifique / Institut National de Physique Nucléaire et de Physique des Particules in France, the Agenzia Spaziale Italiana, the Istituto Nazionale di Fisica Nucleare, and the Istituto Nazionale di Astrofisica in Italy, the Ministry of Education, Culture, Sports, Science and Technology (MEXT), High Energy Accelerator Research Organization (KEK) and Japan Aerospace Exploration Agency (JAXA) in Japan, and the K. A. Wallenberg Foundation and the Swedish National Space Board in Sweden. Additional support for science analysis during the operations phase from the following agencies is also gratefully acknowledged: the Instituto Nazionale di Astrofisica in Italy and the Centre National d'Études Spatiales in France.

Marianne Lemoine-Goumard and Marie-Hélène Grondin acknowledge the ANR PECORA support from the Agence Nationale de la Recherche (ANR-17-CE31-0014).

\section{References}

[1] Abramowski, A., Aharonian, F., et al., 2014a, ApJ, 794, L1

[2] Kothes, R., Dougherty, S. M., 2007, AA, 468, 993

[3] Abramowski, A., Aharonian, F. et al., 2014b, MNRAS, 439, 2828

[4] Whiteoak, J. B. Z. \& Green, A. J., 1996, AAS, 118, 329

[5] Gotthelf, E. V., Tomsick, J. A., Halpern, J. P., et al., 2014, ApJ, 788, 155

[6] Lemoine-Goumard, M., Grondin, M.-H., et al., 2014, ApJ, 794, L16

[7] Atwood, W. B. et al, 2009, ApJ, 697, 1071

[8] Atwood, W. B. et al., 2013, arXiv:1303.3514 Bruel, P. et al, 2018, arXiv:1810.11394

[9] Rochester, L. et al, 2009, arXiv:1001.5005

[10] Wood, M., Caputo, R., 2017, proceedings of the 35th ICRC, arxiv:1707.09551

[11] Abdo, A. A. et al. 2009, Phys. Rev. D, 80, 122004

[12] The Fermi-LAT collaboration, 2019, arXiv:1902.10045 
[13] Mattox, J. R., Bertsch, D. L., Chiang, J. et al., 1996, ApJ, 461, 396

[14] Acero, F. et al., 2016, ApJS, 224,8 